\title{
Article \\ Serum Dickkopf-1 in Combined with CA 19-9 as a Biomarker of Intrahepatic Cholangiocarcinoma
}

\author{
Si-Young Kim ${ }^{1,+} \oplus$, Hee-Seung Lee ${ }^{1,+}{ }^{\text {, Seung-Min Bang }}{ }^{1}$, Dai-Hoon Han ${ }^{2}{ }^{\circledR}$, Ho-Kyoung Hwang ${ }^{2}$, \\ Gi-Hong Choi ${ }^{2}$, Moon-Jae Chung ${ }^{1, *}$ and Seung-Up Kim ${ }^{1,3, *(D)}$ \\ 1 Department of Internal Medicine, Yonsei University College of Medicine, Seoul 120-752, Korea; \\ kimsy0616@naver.com (S.-Y.K.); LHS6865@yuhs.ac (H.-S.L.); bang7028@yuhs.ac (S.-M.B.) \\ 2 Department of Hepatobiliary and Pancreatic Surgery, Yonsei University College of Medicine, \\ Seoul 120-752, Korea; dhhan@yuhs.ac (D.-H.H.); drhhk@yuhs.ac (H.-K.H.); CHOIGH@yuhs.ac (G.-H.C.) \\ 3 Yonsei Liver Center, Severance Hospital, Seoul 120-752, Korea \\ * Correspondence: MJCHUNG@yuhs.ac (M.-J.C.); ksukorea@yuhs.ac (S.-U.K.); Tel.: +82-2-2228-8473 (M.-J.C.); \\ +82-2-2228-1944 (S.-U.K.); Fax: +82-2-312-9538 (M.-J.C.); +82-2-393-6884 (S.-U.K.) \\ + Si Young Kim and Hee Seung Lee contributed equally to this work.
}

check for updates

Citation: Kim, S.-Y.; Lee, H.-S.; Bang, S.-M.; Han, D.-H.; Hwang, H.-K.; Choi, G.-H.; Chung, M.-J.; Kim, S.-U. Serum Dickkopf-1 in Combined with CA 19-9 as a Biomarker of Intrahepatic Cholangiocarcinoma Cancers 2021, 13, 1828. https://doi.org/10.3390/ cancers13081828

Academic Editor: Kevin Gaston

Received: 26 February 2021

Accepted: 8 April 2021

Published: 12 April 2021

Publisher's Note: MDPI stays neutral with regard to jurisdictional claims in published maps and institutional affiliations.

Copyright: (c) 2021 by the authors. Licensee MDPI, Basel, Switzerland. This article is an open access article distributed under the terms and conditions of the Creative Commons Attribution (CC BY) license (https:// creativecommons.org/licenses/by/ $4.0 /)$.
Simple Summary: Cholangiocarcinoma (CCC) is a rare cancer, but its incidence and mortality have been increased in the past few decades worldwide, representing a global health problem. CCC is usually asymptomatic in early stages and, therefore, often diagnosed when the disease is already in advanced stages, which highly compromises therapeutic options, resulting in a dismal prognosis. The current diagnosis of CCC by non-invasive approaches such as serum biomarker, carbohydrate antigen 19-9 (CA 19-9), is not accurate enough due to the limitations in its low sensitivity, especially at the early stages of the disease. Therefore, new biomarkers with higher sensitivity and specificity are needed. As the clinical significance of dickkopf-related protein-1 (DKK-1) has been reported in various tumors including intrahepatic CCC (ICC), we aimed to identify the diagnostic and prognostic performance of the DKK-1 and its additive effect combined with CA 19-9 in patients with CCC.

Abstract: Dickkopf-related protein 1 (DKK-1) has a diagnostic and prognostic value in various malignant tumors. We investigated the diagnostic and prognostic performance of DKK-1 in combination with carbohydrate antigen 19-9 (CA 19-9) in cholangiocarcinoma (CCC) patients. Serum DKK-1 levels were measured using enzyme-linked immunosorbent assay. The receiver operating characteristic (ROC) curve, area under ROC (AUROC) analyses, Kaplan-Meier method, and Cox proportional hazard model were used to evaluate the diagnostic and prognostic performance of DKK-1 in combination with CA 19-9. We checked DKK-1 levels in 356 CCC patients and found that DKK-1 was significantly elevated only in 79 intrahepatic CCC (ICC) patients compared to controls (340.5 vs. $249.8 \mathrm{pg} / \mathrm{mL}, p=0.002$ ). The optimal cutoff level of DKK-1 used to identify ICC patients was $258.0 \mathrm{pg} / \mathrm{mL}$ (AUROC $=0.637$, sensitivity $=59.5 \%$, specificity $=56.9 \%$, positive predictive value $(\mathrm{PPV})=40.5 \%$, negative predictive value $(\mathrm{NPV})=74.0 \%$, positive likelihood ratio $(\mathrm{LR})=1.38$, and negative LR $=0.71)$. Using this cutoff, $47(59.5 \%)$ patients were correctly diagnosed with ICC. DKK-1 in combination with CA 19-9 showed a better diagnostic performance (AUROC $=0.793$, sensitivity $=74.7 \%$, specificity $=56.3 \%$, $\mathrm{PPV}=45.7, \mathrm{NPV}=81.8$, positive $\mathrm{LR}=1.71$, and negative $\mathrm{LR}=0.45$ ) than CA 19-9 alone. The low DKK-1 and CA 19-9 expression group had a significantly longer overall survival (OS) than the high expression group $(p=0.006)$. The higher level of DKK-1 and CA 19-9 was independently associated with shorter OS (hazard ratio $=3.077,95 \%$ confidence interval 1.389-6.819, $p=0.006$ ). The diagnostic and prognostic performance of DKK-1 in combination with CA 19-9 might be better than those of CA 19-9 alone in ICC patients.

Keywords: dickkopf-related protein 1; carbohydrate antigen 19-9; intrahepatic cholangiocarcinoma; diagnosis 


\section{Introduction}

Cholangiocarcinoma (CCC) is an epithelial cell malignancy arising from different locations in the biliary tree. It can be classified into intrahepatic, perihilar, and distal cholangiocarcinomas [1]. The prognosis of CCC patients is poor because most patients are diagnosed at an advanced stage [2].

There is no blood biomarker with high accuracy for CCC [3]. The serum levels of known biomarkers such as carcinoembryonic antigen and carbohydrate antigen 19-9 (CA 19-9) are measured to diagnose $C C C$, but these biomarkers have limitations due to their low sensitivity, especially at the early stages of the disease [3]. Clinicians use CA 19-9 for prognosis prediction, but the false-positive rate is high because of cholangitis [4]. Therefore, new biomarkers with higher sensitivity and specificity are needed.

Dickkopf-related protein 1 (DKK-1) is a member of the Dickkopf family that was identified as a secreted protein and is an inhibitor of Wnt/ $\beta$-catenin signaling pathway $[5,6]$. The clinical and prognostic significance of DKK-1 has been reported in breast cancer, lung cancer, esophageal cancer, myeloma, pancreatic cancer, and hepatocellular carcinoma [7-14]. Shi et al. reported that DKK-1 was highly expressed in intrahepatic CCC (ICC) tumor tissues after surgery, and the serum DKK-1 level was significantly higher than that of the control group, indicating a potential role of DKK-1 as a diagnostic surrogate for ICC patients [15].

We investigated the diagnostic and prognostic performance of DKK- 1 and its additive effect when combined with CA 19-9 in ICC patients.

\section{Materials and Methods}

\subsection{Blood Samples and Clinical Information}

Blood samples and data used in this study were obtained from the Severance Hospital Biobank and the Korea Biobank Network (The Biobank of Pusan National University Hospital, Pusan, Korea, Gyeongsang National University Hospital, Jinju, Korea, Keimyung University Dongsan Hospital, Daegu, Korea, Chonbuk National University Hospital, Jeonju, Korea, Chungnam National University Hospital, Daejeon, Korea, and Korea Institute of Radiological and Medical Sciences Radiation Biobank, Seoul, Korea, which is affiliated with Korea Cancer Center Hospital, Seoul, Korea, are members of Korea Biobank Network, Cheongju, Korea).

Ninety-two blood samples from CCC patients who underwent surgery were collected from Severance Hospital, Yonsei University, Republic of Korea, between April 2012 and April 2017. Blood samples were obtained prior to surgical treatment and stored at $-80{ }^{\circ} \mathrm{C}$ in the Severance Hospital Biobank. An additional 264 blood samples from CCC patients and 200 blood samples from healthy individuals were collected from the Korea Biobank Network of hospitals. Blood samples from CCC patients were collected from patients who underwent surgery at Pusan National University Hospital, Pusan, Korea, Gyeongsang National University Hospital, Jinju, Korea, Keimyung University Dongsan Hospital, Daegu, Korea, and Chonbuk National University Hospital, Jeonju, Korea $(n=84,80,50$, and 50, respectively) between February 2011 and February 2019.

Further, blood samples were obtained from healthy individuals who visited the healthcare center of Chonbuk National University Hospital, Jeonju, Korea, Chungnam National University Hospital, Daejeon, Korea, and Korea Cancer Center Hospital, Seoul, Korea ( $n=50,50$, and 100, respectively) for a health screening check-up between January 2015 and December 2018. Healthy individuals included male and female subjects aged 20-70 years with no past or current clinical abnormalities such as malignancies. Blood samples from the Korea Biobank Network were collected using BD Vacutainer SS Plus Blood Collection Tubes (BD Biosciences, San Jose, CA, USA) from CCC patients prior to surgery and during a health screening check-up involving healthy individuals. Whole blood samples were centrifuged at $3000 \mathrm{rpm}$ at $4{ }^{\circ} \mathrm{C}$ for $20 \mathrm{~min}$, and the supernatant was immediately apportioned into $500 \mu \mathrm{L}$ aliquots and stored at $-80^{\circ} \mathrm{C}$ in each biobank. 
Clinical data and patient characteristics were obtained from electronic medical records of each hospital. Therefore, some clinical information was only available at Severance Hospital. In the case of tumor stage, tumor-node-metastasis staging based on the staging classification of the American Joint Commission on Cancer (AJCC, 8th edition) was assessed postoperatively. Since all patients in this study received surgical treatment, the surgical method was assessed and divided into major (hepatectomy, extended hepatectomy) and minor (wedge resection, sectionectomy, bisectionectomy) liver resection according to extent of anatomical resection [16].

\subsection{Patient Selection}

We checked DKK-1 levels in 356 CCC patients and 200 healthy individuals. Since the median DKK-1 levels in healthy individuals and CCC patients were $249.8 \mathrm{pg} / \mathrm{mL}$ and $267.2 \mathrm{pg} / \mathrm{mL},(p=0.077)$ respectively, and given the known association of DKK-1 with ICC, we selected the ICC $(n=79)$ group to further analysis. The ICC group had a significantly higher DKK-1 level than the ECC group (median: $340.5 \mathrm{pg} / \mathrm{mL}$ vs. $255.0 \mathrm{pg} / \mathrm{mL}$, $p=0.033$ ) and healthy population (median: $340.5 \mathrm{pg} / \mathrm{mL}$ vs. $249.8 \mathrm{pg} / \mathrm{mL}, p=0.002$ ). There was no significant difference between the ECC group and healthy population (median: $255.0 \mathrm{pg} / \mathrm{mL}$ vs. $249.8 \mathrm{pg} / \mathrm{mL}, p=0.377)$. We, therefore, studied ICC to determine the diagnostic and predictive role of DKK-1 in CCC (Figure S1A,B).

Among the 79 ICC patients, 20 were patients from the Severance Hospital, and the remaining 59 were patients from other hospitals. As a control group, 160 out of 200 healthy individuals were randomly selected corresponding to two times the number of ICC patients (Figure 1).

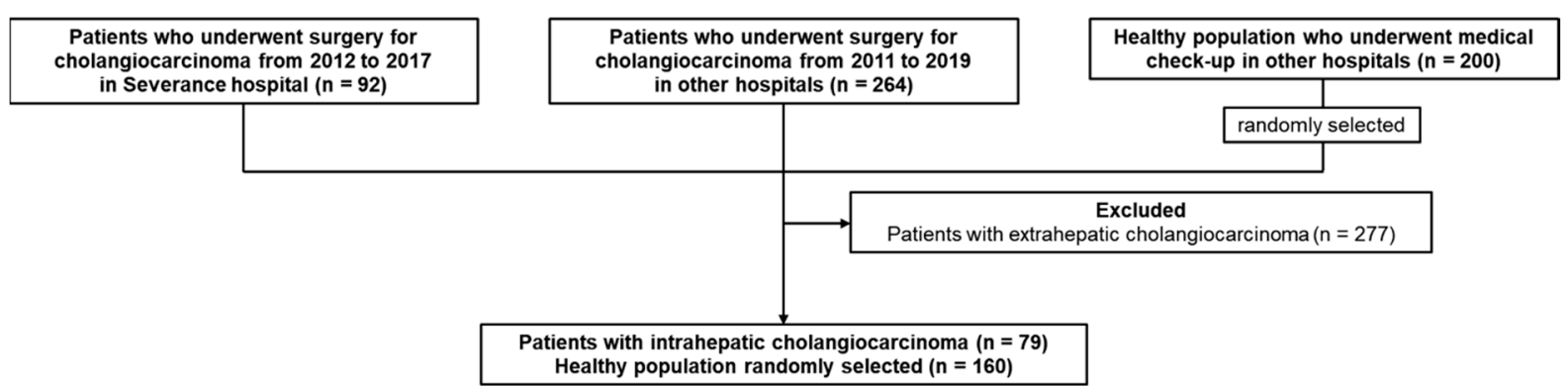

Figure 1. Study flowchart. We collected blood samples of 92 patients with CCC from the Severance hospital. Two hundred and sixty-four blood samples of patients with CCC and 200 blood samples of healthy individuals were collected from other hospitals in the Korea Biobank Network. A total of 277 patients with ECC were excluded and 79 patients with ICC were remained. Of the total 200 healthy individuals, 160 were randomly selected corresponding to two times the number of ICC patients.

\subsection{Measurement of DKK-1 and CA 19-9}

DKK-1 levels were measured using the enzyme-linked immunosorbent assay (ELISA) method (human DKK-1 DuoSet ELISA kit). ELISA was performed according to the manufacturer's instructions (R\&D Systems, Minneapolis, MN, USA). Binding of DKK-1 to its receptor (LRP-6) was measured using a functional ELISA. Briefly, microtiter plates were coated with $3 \mu \mathrm{g} / \mathrm{mL}$ of human LRP-6-Fc chimera (recombinant human LRP6/Fc chimera; $\mathrm{R} \& \mathrm{D}$ Systems) prior to the addition of serum samples, and detection was performed using human anti-DKK-1 antibody (R \& D Systems). CA 19-9 was measured with the VITROS 3600 Immunodiagnostic System (Ortho-Clinical Diagnostics Inc., Raritan, NJ, USA) using a chemiluminescence immunoassay.

\subsection{Statistical Analyses}

The Mann-Whitney U and Kruskal-Wallis tests were used for comparisons between groups. Receiver operating characteristic (ROC) analysis was used to determine the diagnostic potential of DKK-1, CA 19-9, and a combination of the two markers. To assess 
whether the combination of DKK-1 and CA 19-9 was better than each marker, we created a new variable predicted probability by using a binary logistic regression equation. Survival curves were estimated using the Kaplan-Meier method, and the differences among curves were assessed using log-rank tests. Univariable and multivariable analyses were based on the Cox proportional hazards regression model for risk factors influencing survival in patients. A $p$-value $<0.05$ was considered statistically significant. We set the cutoff value for CA $19-9$ as $37.0 \mathrm{U} / \mathrm{mL}$, which is the standard cutoff value [17]. The diagnostic cutoff value for DKK-1 was calculated using the coordinate values of the ROC curve. To determine the optimal cutoff value, we used the criterion for determining the point where the sensitivity is equal to the specificity. This point on the curve is where the product of sensitivity and specificity is maximum [18,19]. Statistical analyses were performed using the SPSS software package (version 20.0; IBM, Armonk, NY, USA). The statistical significance between the ROC curves and the area under the ROC curves (AUROCs) were calculated using MedCalc version 11.1 (MedCalc Software, Mariakerke, Belgium).

\section{Results}

\subsection{Patient Characteristics}

The mean age of the 79 ICC patients was 67.3 years, and the proportion of male participants was $70.9 \%(n=56)$. Diabetes and hypertension were identified in $11(55 \%)$ and $15(75 \%)$ patients, respectively. AJCC stages I, II, III and IV were observed in 18 (39.1\%), $9(19.6 \%), 12(26.1 \%)$, and $7(15.2 \%)$ patients, respectively. The median levels of aspartate aminotransferase (AST), alanine aminotransferase (ALT), and total bilirubin were $41.0 \mathrm{U} / \mathrm{L}$, $41.5 \mathrm{U} / \mathrm{L}$, and $1.01 \mathrm{mg} / \mathrm{dL}$, respectively. The median levels of DKK-1 and CA 19-9 were $381.1 \mathrm{pg} / \mathrm{mL}$ and $38.2 \mathrm{U} / \mathrm{mL}$, respectively (Table 1 ).

Table 1. Baseline characteristics.

\begin{tabular}{cc}
\hline Variables & Patients $(\boldsymbol{n}=\mathbf{7 9 )}$ \\
\hline Male gender & $56(70.9)$ \\
Age (years) & $67.3 \pm 11.0$ \\
Diabetes mellitus * & $11(55.0)$ \\
Hypertension * & $15(75.0)$ \\
Smoking * & \\
Never/ current or ex & $11(55.0) / 9(45.0)$ \\
Alcohol * & $12(60.0) / 8(40.0)$ \\
Never/ current or ex & \\
AJCC stage * & $18(39.1)$ \\
I & $9(19.6)$ \\
II & $12(26.1)$ \\
III & $7(15.2)$ \\
AST $(\mathrm{U} / \mathrm{L})$ & $41.0(24.8-147.8)$ \\
ALT $(\mathrm{U} / \mathrm{L})$ & $41.5(21.8-102.0)$ \\
Total bilirubin $(\mathrm{mg} / \mathrm{dL})$ & $1.01(0.46-3.14)$ \\
DKK-1 $(\mathrm{pg} / \mathrm{mL})$ & $340.5(206.9-587.2)$ \\
CA 19-9 $(\mathrm{U} / \mathrm{mL})$ & $32.0(12.6-193.7)$ \\
\hline
\end{tabular}

Variables are expressed as mean \pm standard deviation, median (interquartile range), or $n(\%)$. ${ }^{*}$ Patients without clinical information were not included. AJCC, American Joint Commission on Cancer; DKK-1, Dickkopf-related protein 1; CA 19-9, carbohydrate antigen 19-9; AST, aspartate aminotransferase; ALT, alanine aminotransferase.

\subsection{Stratification of Cholangiocarcinoma by Etiologies}

We stratified CCC patients by its possible etiologies for further analysis. Of the total 356 CCC patients, 95 patients were able to identify the etiology, and they were classified into sporadic $(n=75)$, hepatitis B virus (HBV) $(n=5)$, hepatitis C virus (HCV) $(n=1)$, liver fluke $(n=1)$, liver cirrhosis $(n=6)$, PSC $(n=0)$, and bile duct stone $(n=7)$, respectively, and their median level of DKK-1 and CA 19-9 are described in Table S1. 


\subsection{Diagnostic Performance of DKK-1 and CA 19-9}

To identify diagnostic performance of DKK-1, CA 19-9, and a combination of the two markers, we checked ROC curves and area under the ROC (AUROC) of them. The AUROCs of DKK-1 and CA 19-9 were 0.637 and 0.769, respectively, and when the two markers were combined, the AUROC was 0.793, which was higher than that of the CA 19-9 alone (Figure 2).

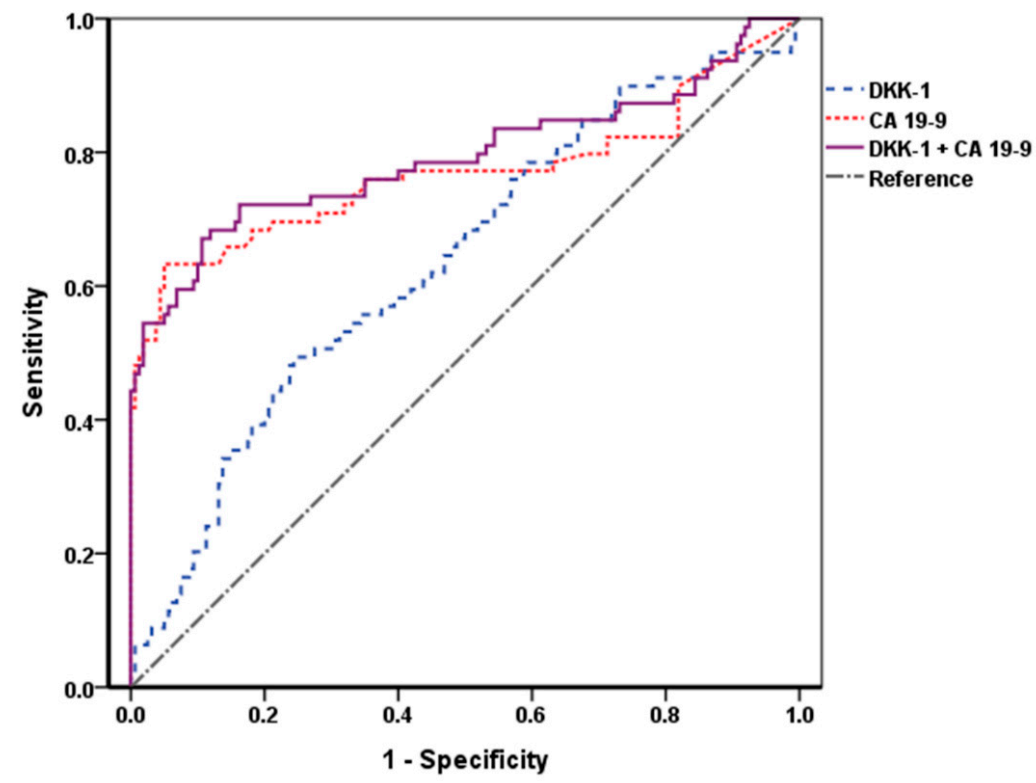

Figure 2. ROC curves of DKK-1, CA 19-9, and their combination. The AUROCs of DKK-1 and CA 19-9 were 0.637 and 0.769 , respectively, and the AUROC when the two markers were combined was 0.793. When combined with DKK-1, the AUROC was higher than that of the CA 19-9 alone. DKK-1, Dickkopf-related protein 1; CA 19-9, carbohydrate antigen 19-9; ROC, receiver operating characteristic; AUROC, area under the ROC.

The calculated cutoff value for DKK-1 was $258.0 \mathrm{pg} / \mathrm{mL}$, and that for CA 19-9 was set as $37.0 \mathrm{U} / \mathrm{mL}$, which is widely accepted [17]. The diagnostic indices of DKK-1, CA 19-9, and their combination are described in Table S2. The sensitivity and negative predictive value (NPV) were higher when DKK-1 and CA 19-9 were combined compared to CA 19-9 alone. (sensitivity $=74.7 \%$ vs. $49.4 \%$ and NPV $=81.8 \%$ vs. $79.8 \%$ ).

\subsection{DKK-1 in CA 19-9 False-Negative Patients}

To determine the additive effect of DKK-1 on ICC diagnosis, the diagnostic yield of DKK-1 was verified. As a result, 20 out of 40 patients with false-negative CA 19-9 were additionally diagnosed with high DKK-1 levels. The diagnostic yield of DKK-1 was 50\% (Figure 3).

\subsection{Prognostic Performance of DKK-1 and CA 19-9}

ICC patients were classified into the high and low expression groups based on a DKK1 level of $258.0 \mathrm{pg} / \mathrm{mL}$ and a CA $19-9$ level of $37.0 \mathrm{U} / \mathrm{mL}$, respectively. The median survival of the low and high DKK- 1 expression groups were 41.7 and 28.0 months, respectively, and there was no significant difference between the groups ( $p=0.231$ by log-rank test). The median survival of the low and high CA 19-9 expression groups were 53.0 and 27.5 months, respectively, and there was no significant difference between the groups $(p=0.117$ by logrank test) (Figure S2A,B). 


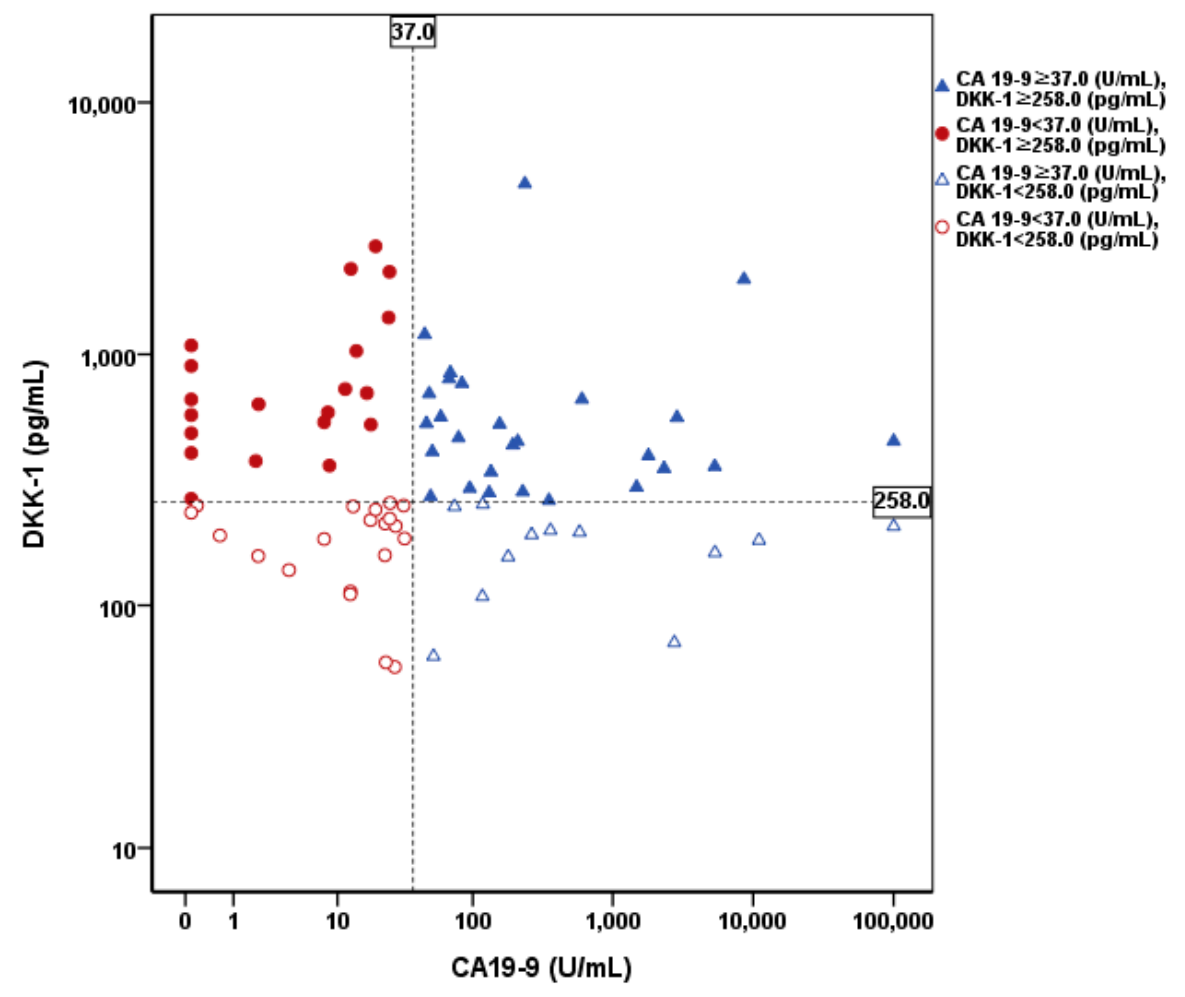

Figure 3. Distribution of DKK-1 and CA 19-9 levels in patients with ICC. The cutoff value of DKK-1 and CA 19-9 were $258.0 \mathrm{pg} / \mathrm{mL}$ and $37.0 \mathrm{U} / \mathrm{mL}$, respectively. Twenty out of 40 patients with false negative CA 19-9 were additionally diagnosed using DKK-1. The diagnostic yield of DKK-1 was 50\%. DKK-1, Dickkopf-related protein 1; CA 19-9, carbohydrate antigen 19-9; ICC, intrahepatic cholangiocarcinoma.

To further identify the prognostic performance of DKK-1 and CA 19-9 separately, a Cox proportional hazard model analysis was performed. The hazard ratio of DKK-1 and CA 19-9 were 1.408 (95\% CI 0.802-2.472, $p=0.234)$ and 1.542 (95\% CI 0.893-2.660, $p=0.120)$ in univariate analysis and these results were not statistically significant (Table 2 ).

Table 2. Univariate and multivariate analysis to identify independent predictors for survival in patients with ICC.

\begin{tabular}{|c|c|c|c|c|}
\hline \multirow{2}{*}{ Variables } & \multicolumn{2}{|c|}{ Univariate } & \multicolumn{2}{|c|}{ Multivariate } \\
\hline & HR $(95 \%$ CI $)$ & $p$ Value & HR $(95 \%$ CI $)$ & $p$ Value \\
\hline DKK-1 $\geq 258.0$ pg $/ \mathrm{mL}$ (vs. DKK-1 < 258.0 pg $/ \mathrm{mL}$ ) & $1.408(0.802-2.472)$ & 0.234 & & \\
\hline CA $19-9 \geq 37.0 \mathrm{U} / \mathrm{mL}$ (vs. CA $19-9<37.0 \mathrm{U} / \mathrm{mL}$ ) & $1.542(0.893-2.660)$ & 0.120 & & \\
\hline $\begin{array}{c}\text { DKK-1 } \geq 258.0 \mathrm{pg} / \mathrm{mL} \text { or CA } 19-9 \geq 37.0 \mathrm{U} / \mathrm{mL} \\
(\text { vs. DKK- } 1<258.0 \mathrm{pg} / \mathrm{mL} \text { and CA } 19-9<37.0 \mathrm{U} / \mathrm{mL})\end{array}$ & $2.753(1.294-5.858)$ & 0.009 & $3.077(1.389-6.819)$ & 0.006 \\
\hline Age & $1.020(0.992-1.048)$ & 0.160 & $1.010(0.981-1.039)$ & 0.511 \\
\hline Female gender & $0.922(0.510-1.664)$ & 0.787 & $1.287(0.654-2.535)$ & 0.465 \\
\hline Stage III and IV (vs. I and II) * & $1.828(0.880-3.800)$ & 0.106 & & \\
\hline Major surgery (vs. minor surgery) ${ }^{*, \dagger}$ & $1.476(0.469-4.648)$ & 0.506 & & \\
\hline AST $(\mathrm{IU} / \mathrm{L})$ & $1.000(0.998-1.003)$ & 0.794 & & \\
\hline ALT (IU/L) & $1.000(0.997-1.003)$ & 0.904 & & \\
\hline Total bilirubin (mg/dL) & $1.096(1.032-1.165)$ & 0.003 & $1.084(1.019-1.154)$ & 0.011 \\
\hline
\end{tabular}

Variables are expressed as median (interquartile range). * Patients without clinical information were not included. ${ }^{\dagger}$ Major surgery includes hepatectomy and extended hepatectomy, minor surgery includes sectionectomy, bisectionectomy, and wedge resection. ICC, intrahepatic cholangiocarcinoma; DKK-1, Dickkopf-related protein 1; CA 19-9, carbohydrate antigen 19-9; AST, aspartate aminotransferase; ALT, alanine aminotransferase; HR, hazard ratio; $\mathrm{CI}$, confidence interval. 


\subsection{Combination of DKK-1 and CA 19-9}

Since DKK-1 alone did not yield significant results, we checked whether combining DKK-1 with CA 19-9 could predict the prognosis of ICC patients. ICC patients were classified into high expression and low expression groups based on the cutoff values for DKK-1 and CA 19-9. Among the ICC patients, those with both DKK-1 and CA 19-9 levels lower than the cutoff value were assigned to the low expression group, and those with either the DKK-1 or CA 19-9 level higher than the cutoff value were included in the high expression group. Patients in the low expression group had a longer overall survival than those in the high expression group and showed significant difference. (57.5 vs. 27.5 months, $p=0.006$, log-rank test) (Figure 4).

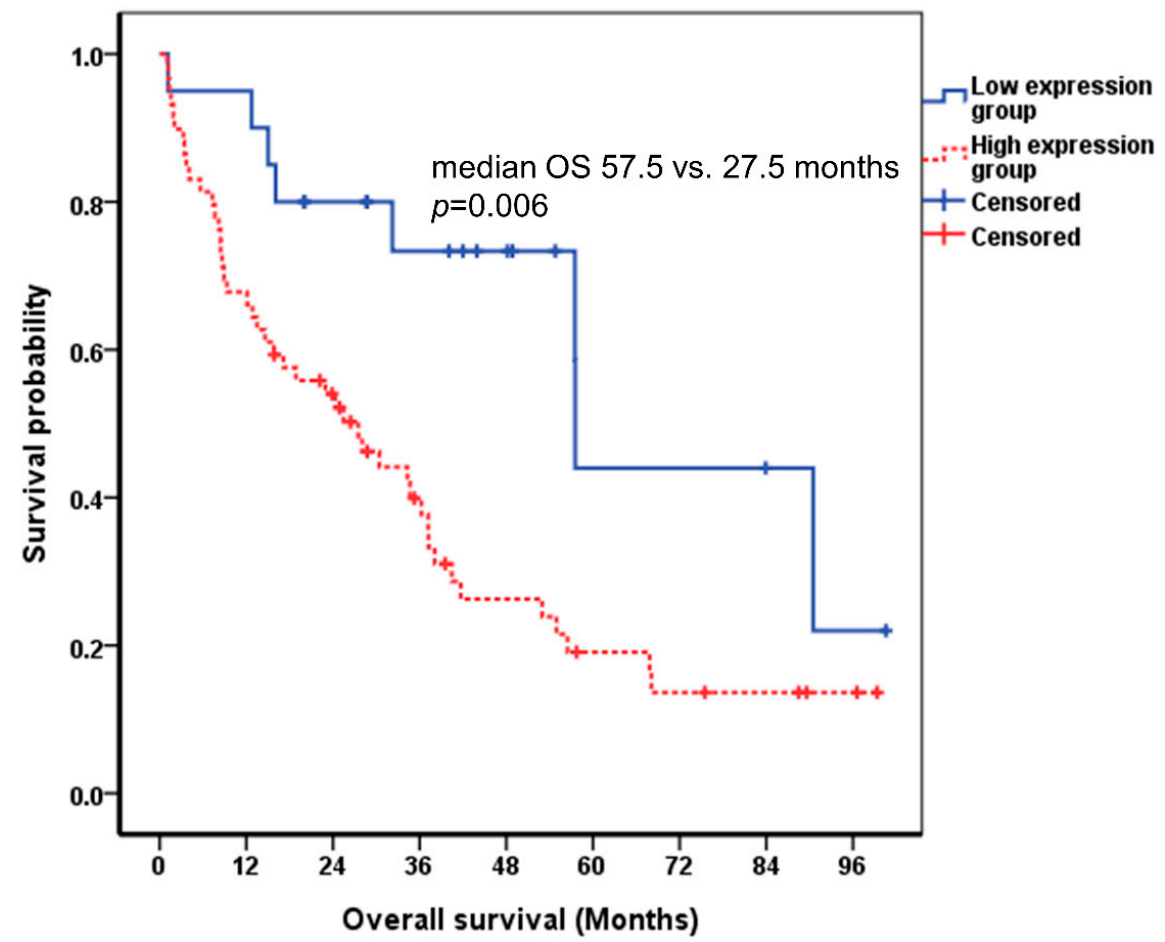

Figure 4. Correlation between DKK-1 combined with CA 19-9 and the survival of patients with ICC using the Kaplan-Meier curve. The cutoff value of DKK-1 and CA $19-9$ were $258.0 \mathrm{pg} / \mathrm{mL}$ and $37.0 \mathrm{U} / \mathrm{mL}$, respectively. The median survival of the low and high expression groups were 57.5 and 27.5 months, respectively ( $p=0.006$ by log-rank test). DKK-1, Dickkopf-related protein 1; CA 19-9, carbohydrate antigen 19-9; ICC, intrahepatic cholangiocarcinoma; OS, overall survival.

In the Cox proportional hazard model analysis, the higher level of DKK-1 and CA 19-9 was independently associated with shorter survival (HR $=3.077,95 \%$ CI $1.389-6.819$, $p=0.006)$, together with higher level of total bilirubin (HR $=1.084,95 \%$ CI 1.019-1.154, $p=0.011)$ (Table 2).

\section{Discussion}

To date, no blood biomarkers with sufficient diagnostic and prognostic accuracy have been identified in ICC patients. We observed that a novel serum biomarker, DKK-1, when combined with a conventional serum biomarker, CA 19-9, exhibited acceptable diagnostic and prognostic performance, especially in ICC patients. To the best of our knowledge, this is the largest study to investigate the clinical implications of serum DKK-1 in ICC patients.

Although serum DKK-1 has been reported as a useful biomarker in several cancers, its clinical implications are controversial. For example, serum DKK-1 levels are high in patients with cervical, endometrial cancer, and hepatocellular carcinoma, when compared to controls [20-22]. In contrast, some studies have shown that DKK-1 expression is downregulated in colorectal cancer, brain tumor, and papillary thyroid cancer [23-25]. In spite 
of these controversies, it has been reported that high DKK-1 levels are significantly associated with CCC. Shi et al. showed that serum DKK-1 level is positively correlated with lymphatic metastasis and is indicative of poor prognosis during ICC, and noted that the optimal cutoff value of DKK-1 for detecting CCC was $2490 \mathrm{pg} / \mathrm{mL}$ (sensitivity $=75.7 \%$ and specificity $=100 \%$ ) [15]. Liu et al. reported that a higher expression of DKK-1 in hilar CCC is significantly correlated with hilar lymph node metastasis, and noted that a loss of DKK-1 expression significantly inhibited cancer cell proliferation and migration [26].

Due to the insufficient diagnostic and prognostic performance of single biomarkers such as CA 19-9, attempts to combine multiple serum biomarkers to enhance the overall diagnostic or prognostic performance have been made in several cancers including CCC $[22,27,28]$. In CCC, serum matrix metalloproteinase-7, soluble fragment of cytokeratin19, and miR-1537 combined with CA 19-9 showed higher diagnostic performance than CA 19-9 alone [29-31]. Similarly, we showed that the AUROC, sensitivity, and NPV of the combined use of DKK-1 and CA 19-9 was higher than that of CA 19-9 alone. Additionally, we found that DKK-1 can identify 50\% ICC patients among those with normal CA 19-9 levels. These findings might support the diagnostic performance of serum DKK-1 in ICC patients.

Although the calculated cutoff level of DKK-1 in our study $(258.0 \mathrm{pg} / \mathrm{mL})$ was similar to or higher than those of other cancers, such as cervical $(314.13 \mathrm{pg} / \mathrm{mL})$, endometrial $(46.95 \mathrm{pg} / \mathrm{mL})$, and gastric $(31.92 \mathrm{pg} / \mathrm{mL})$ cancers [21,32], it was much lower than the cutoff proposed for ICC in previous studies $(2490 \mathrm{pg} / \mathrm{mL})$ [15]. The exact reason for the difference in DKK-1 cutoff value is unclear; however, it may be because all the patients in this study, including those in the validation cohort whose tumor stage was not provided, underwent surgical treatment, which means that they were in the early stage of the disease. It may also be because of different etiologies of the CCC [33]. Some studies have shown that sporadic cholangiocarcinoma is related to the $\mathrm{Wnt} / \beta$-catenin signaling pathway associated with DKK-1, whereas primary sclerosing cholangitis associated cholangiocarcinoma is primarily related to inflammation-related cytokine and chemokine pathways [34-36]. Therefore, further research is needed to establish an optimal DKK-1 cutoff value.

Interestingly, there was no significant association between DKK-1 and ECC in terms of prognosis or diagnosis in our study. While FGFR2 fusions are commonly found in ICC, KRAS mutations are frequent in ECC and gallbladder cancer and significantly associated with poor prognosis. This mutational difference may be related to the difference in DKK-1 levels between biliary tract cancers [37]. In another aspect, embryologically, intrahepatic cholangiocytes arise from bipotent hepatoblasts, and extrahepatic cholangiocytes share an embryologic origin with the ventral pancreas [38]. Rimland et al. reported that cells from intrahepatic and extrahepatic biliary trees could be diverged in response to the canonical Wnt pathway, which is associated with DKK-1. To explain in more detail, they showed that canonical Wnt signaling caused intrahepatic bile duct cells to cease proliferation whereas extrahepatic bile duct cells could be grown over a prolonged period by an experiment using organoid [39]. In addition, Chen et al. revealed that the canonical Wnt pathway was more activated in ECC than in ICC by confirming the protein level in tissue samples [40]. These recent studies show that the environment of intrahepatic bile duct with relatively less activated canonical Wnt pathway may be related to the increased expression of DKK-1. Therefore, the reason why DKK-1 has different associations with ICC and ECC is probably due to the embryologic and carcinogenetic mutational differences. Further experimental studies will be needed in the future and we would like to propose a prospective study on the diagnostic and prognostic efficacy of DKK-1 in CA 19-9 negative biliary tract cancer patients based on this study for clinical development.

We are also aware of the limitations of our study. First, our study was a retrospective study, which led to a potential selection bias. Specifically, patients with relatively early stage CCC who underwent surgical resection might have been recruited. However, we validated our main results using blood samples from ICC patients and healthy individuals from other hospitals that belong to the Korea Biobank Network to minimize the probability of selection bias. Second, there were restrictions on collecting sufficient clinical information 
for the healthy subjects due to sample acquisition from the biobanks of various hospitals. Since no other co-variables except serum DKK-1 and CA 19-9 were available in the study population, sufficient adjustment for confounders was not performed. Third, the relatively small number of recruited ICC patients from different institutions might be another pitfall of this study. For this reason, it was difficult to access unified and consistent information in clinical data, especially for tumor stage and comorbid diseases, obtained from various hospitals. However, this was necessary to increase the number of patients, as ICC itself is a rare malignancy [41].

\section{Conclusions}

In conclusion, we showed that serum DKK-1 levels are relatively higher in ICC patients than in ECC patients and healthy individuals. The combined use of DKK-1 and CA 19-9 increased the sensitivity and NPV for discriminating ICC patients from healthy individuals. Additionally, combining two markers could help predict the prognosis of ICC patients. Further large-scale studies are warranted to validate our results.

Supplementary Materials: The following are available online at https://www.mdpi.com/article/ 10.3390 / cancers13081828/s1, Figure S1. Serum DKK-1 level. (A) The median level of DKK-1 in the control group $(n=200)$ and in patients with CCC $(n=356)$ were $249.8 \mathrm{pg} / \mathrm{mL}$ and $267.2 \mathrm{pg} / \mathrm{mL}$, respectively. ( $p=0.077)$ (B) When patients with CCC were divided into the ECC group $(n=277)$ and ICC group ( $n=79$ ), the median levels of DKK-1 were $255.0 \mathrm{pg} / \mathrm{mL}$ and $340.5 \mathrm{pg} / \mathrm{mL}$, respectively, and there was a significant difference between the control group and the ICC group. $(p=0.002)$ DKK-1, Dickkopf-related protein 1; CCC, cholangiocarcinoma; ECC, extrahepatic cholangiocarcinoma; ICC, intrahepatic cholangiocarcinoma; Figure S2. Correlation between DKK-1 and CA 19-9 level and the survival of patients with ICC using the Kaplan-Meier curve. The cutoff value of DKK-1 and CA 19-9 were $258.0 \mathrm{pg} / \mathrm{mL}$ and $37.0 \mathrm{U} / \mathrm{mL}$, respectively. (A) The median survival of the low and high DKK-1 expression groups were 41.7 and 28.0 months, respectively, and there was no significant difference between the groups ( $p=0.231$ by log-rank test). (B) The median survival of the low and high CA 19-9 expression groups were 53.0 and 27.5 months, respectively, and there was no significant difference between the groups ( $p=0.117$ by log-rank test). DKK-1, Dickkopf-related protein 1; CA 19-9, carbohydrate antige 19-9; ICC, intrahepatic cholangiocarcinoma; OS, overall survival; Table S1. Etiologies of cholangiocarcinoma and serum levels of biomarkers; Table S2. Performance characteristics of DKK-1 and CA 19-9.

Author Contributions: S.-U.K. and M.-J.C. designed this study; S.-Y.K. and H.-S.L. carried out the data analysis and drafted the manuscript; S.-Y.K., D.-H.H., H.-K.H. and G.-H.C. collected samples and data; H.-S.L., S.-M.B., S.-U.K. and M.-J.C. contributed analysis of samples; All authors have read and agreed to the published version of the manuscript.

Funding: This study was supported by Basic Science Research Program through the National Research Foundation of Korea (NRF), Daejeon, Korea, funded by the Ministry of Science, ICT \& Future Planning, Sejong, Korea (2019R1A2C4070136) and the 2019 Yonsei University College of Medicine Medical Student Research Fund. This study was supported by the 2020 Yonsei University College of Medicine Research Fund (6-2020-0079). The funders had no role in study design, data collection and analysis, decision to publish, or preparation of the manuscript.

Institutional Review Board Statement: This study was performed in accordance with ethical guidelines of the 1975 Declaration of Helsinki and ethical approval was obtained from the Yonsei University Health System, Severance Hospital, Institutional Review Board (IRB No. 4-2018-0226).

Informed Consent Statement: Given its retrospective nature, written informed consent was not required to access clinical data. All samples derived from the Korea Biobank Network were obtained with informed consent under institutional review board-approved protocols.

Data Availability Statement: Restrictions apply to the availability of these data. Data was obtained from Korea Biobank Network and area available at https:/ / www.koreabiobank.re.kr (23 April 2020) with the permission of Korea Biobank Network. 
Acknowledgments: The biospecimens and data used for this study were provided by the Biobank of Pusan National University Hospital, Pusan, Korea, Gyeongsang National University Hospital, Jinju, Korea, Keimyung University Dongsan Hospital, Daegu, Korea, Chonbuk National University Hospital, Jeonju, Korea, Chungnam National University Hospital, Daejeon, Korea, and Korea Institute of Radiological and Medical Sciences Radiation Biobank, Seoul, Korea, members of the Korea Biobank Network, which is supported by the Ministry of Health, Welfare and Family Affairs, Sejong, Korea. All samples derived from the Korea Biobank Network, Cheongju, Korea, were obtained with informed consent under institutional review board-approved protocols.

Conflicts of Interest: The authors declare no conflict of interest.

\begin{abstract}
Abbreviations
CCC, cholangiocarcinoma; CA 19-9, carbohydrate antigen 19-9; DKK-1, Dickkopf-related protein 1; ICC, intrahepatic cholangiocarcinoma; ECC, extrahepatic cholangiocarcinoma; IRB, Institutional Review Board; AJCC, American Joint Commission on Cancer; ELISA, enzyme-linked immunosorbent assay; OS, overall survival; ROC, receiver operating characteristic; AUROC, area under the ROC; AST, aspartate aminotransferase; ALT, alanine aminotransferase; $\mathrm{HBV}$, hepatitis B virus; $\mathrm{HCV}$, hepatitis $\mathrm{C}$ virus; PPV, positive predictive value; NPV, negative predictive value; LR, likelihood ratio; HR, hazard ratio; $\mathrm{CI}$, confidence interval.
\end{abstract}

\title{
References
}

1. Shaib, Y.H.; Davila, J.A.; McGlynn, K.; El-Serag, H.B. Rising incidence of intrahepatic cholangiocarcinoma in the United States: A true increase? J. Hepatol. 2004, 40, 472-477. [CrossRef] [PubMed]

2. Marrero, J.A. Biomarkers in cholangiocarcinoma. Clin. Liver Dis. 2014, 3, 101-103. [CrossRef]

3. Macias, R.I.R.; Banales, J.M.; Sangro, B.; Muntane, J.; Avila, M.A.; Lozano, E.; Perugorria, M.J.; Padillo, F.J.; Bujanda, L.; Marin, J.J.G. The search for novel diagnostic and prognostic biomarkers in cholangiocarcinoma. Biochim. Biophys. Acta Mol. Basis Dis. 2018, 1864 Pt B, 1468-1477. [CrossRef]

4. Macias, R.I.R.; Kornek, M.; Rodrigues, P.M.; Paiva, N.A.; Castro, R.E.; Urban, S.; Pereira, S.P.; Cadamuro, M.; Rupp, C.; Loosen, S.H.; et al. Diagnostic and prognostic biomarkers in cholangiocarcinoma. Liver Int. 2019, 39, 108-122. [CrossRef]

5. Glinka, A.; Wu, W.; Delius, H.; Monaghan, A.P.; Blumenstock, C.; Niehrs, C. Dickkopf-1 is a member of a new family of secreted proteins and functions in head induction. Nature 1998, 391, 357-362. [CrossRef]

6. Semenov, M.V.; Tamai, K.; Brott, B.K.; Kuhl, M.; Sokol, S.; He, X. Head inducer Dickkopf-1 is a ligand for Wnt coreceptor LRP6. Curr. Biol. 2001, 11, 951-961. [CrossRef]

7. Chamorro, M.N.; Schwartz, D.R.; Vonica, A.; Brivanlou, A.H.; Cho, K.R.; Varmus, H.E. FGF-20 and DKK1 are transcriptional targets of beta-catenin and FGF-20 is implicated in cancer and development. EMBO J. 2005, 24, 73-84. [CrossRef]

8. Forget, M.A.; Turcotte, S.; Beauseigle, D.; Godin-Ethier, J.; Pelletier, S.; Martin, J.; Tanguay, S.; Lapointe, R. The Wnt pathway regulator DKK1 is preferentially expressed in hormone-resistant breast tumours and in some common cancer types. Br. J. Cancer 2007, 96, 646-653. [CrossRef]

9. Qian, J.; Xie, J.; Hong, S.; Yang, J.; Zhang, L.; Han, X.; Wang, M.; Zhan, F.; Shaughnessy, J.D., Jr.; Epstein, J.; et al. Dickkopf-1 (DKK1) is a widely expressed and potent tumor-associated antigen in multiple myeloma. Blood 2007, 110, 1587-1594. [CrossRef]

10. Sato, N.; Yamabuki, T.; Takano, A.; Koinuma, J.; Aragaki, M.; Masuda, K.; Ishikawa, N.; Kohno, N.; Ito, H.; Miyamoto, M.; et al. Wnt inhibitor Dickkopf-1 as a target for passive cancer immunotherapy. Cancer Res. 2010, 70, 5326-5336. [CrossRef] [PubMed]

11. Takahashi, N.; Fukushima, T.; Yorita, K.; Tanaka, H.; Chijiiwa, K.; Kataoka, H. Dickkopf-1 is overexpressed in human pancreatic ductal adenocarcinoma cells and is involved in invasive growth. Int. J. Cancer 2010, 126, 1611-1620. [CrossRef] [PubMed]

12. Tian, E.; Zhan, F.; Walker, R.; Rasmussen, E.; Ma, Y.; Barlogie, B.; Shaughnessy, J.D., Jr. The role of the Wnt-signaling antagonist DKK1 in the development of osteolytic lesions in multiple myeloma. N. Engl. J. Med. 2003, 349, 2483-2494. [CrossRef] [PubMed]

13. Yamabuki, T.; Takano, A.; Hayama, S.; Ishikawa, N.; Kato, T.; Miyamoto, M.; Ito, T.; Ito, H.; Miyagi, Y.; Nakayama, H.; et al. Dikkopf-1 as a novel serologic and prognostic biomarker for lung and esophageal carcinomas. Cancer Res. 2007, 67, $2517-2525$. [CrossRef]

14. Yu, B.; Yang, X.; Xu, Y.; Yao, G.; Shu, H.; Lin, B.; Hood, L.; Wang, H.; Yang, S.; Gu, J.; et al. Elevated expression of DKK1 is associated with cytoplasmic/nuclear beta-catenin accumulation and poor prognosis in hepatocellular carcinomas. J. Hepatol. 2009, 50, 948-957. [CrossRef]

15. Shi, R.Y.; Yang, X.R.; Shen, Q.J.; Yang, L.X.; Xu, Y.; Qiu, S.J.; Sun, Y.F.; Zhang, X.; Wang, Z.; Zhu, K.; et al. High expression of Dickkopf-related protein 1 is related to lymphatic metastasis and indicates poor prognosis in intrahepatic cholangiocarcinoma patients after surgery. Cancer 2013, 119, 993-1003. [CrossRef]

16. Karamarkovic, A.; Doklestic, K. Pre-resectional inflow vascular control: Extrafascial dissection of Glissonean pedicle in liver resections. Hepatobiliary Surg. Nutr. 2014, 3, 227-237. 
17. Kim, H.J.; Kim, M.H.; Myung, S.J.; Lim, B.C.; Park, E.T.; Yoo, K.S.; Seo, D.W.; Lee, S.K.; Min, Y.I. A new strategy for the application of CA19-9 in the differentiation of pancreaticobiliary cancer: Analysis using a receiver operating characteristic curve. Am. J. Gastroenterol. 1999, 94, 1941-1946. [CrossRef]

18. Habibzadeh, F.; Habibzadeh, P.; Yadollahie, M. On determining the most appropriate test cut-off value: The case of tests with continuous results. Biochem. Med. 2016, 26, 297-307. [CrossRef]

19. Unal, I. Defining an Optimal Cut-Point Value in ROC Analysis: An Alternative Approach. Comput. Math. Methods Med. 2017, 2017, 3762651. [CrossRef]

20. Inoue, T.; Tanaka, Y. Novel biomarkers for the management of chronic hepatitis B. Clin. Mol. Hepatol. 2020, 26, 261-279. [CrossRef]

21. Jiang, T.; Wang, S.; Huang, L.; Zhang, S. Clinical significance of serum DKK-1 in patients with gynecological cancer. Int. J. Gynecol. Cancer 2009, 19, 1177-1181. [CrossRef] [PubMed]

22. Shen, Q.; Fan, J.; Yang, X.R.; Tan, Y.; Zhao, W.; Xu, Y.; Wang, N.; Niu, Y.; Wu, Z.; Zhou, J.; et al. Serum DKK1 as a protein biomarker for the diagnosis of hepatocellular carcinoma: A large-scale, multicentre study. Lancet Oncol. 2012, 13, 817-826. [CrossRef]

23. Liu, Z.; Sun, B.; Qi, L.; Li, Y.; Zhao, X.; Zhang, D.; Zhang, Y. Dickkopf-1 expression is down-regulated during the colorectal adenoma-carcinoma sequence and correlates with reduced microvessel density and VEGF expression. Histopathology 2015, 67, 158-166. [CrossRef]

24. Shou, J.; Ali-Osman, F.; Multani, A.S.; Pathak, S.; Fedi, P.; Srivenugopal, K.S. Human Dkk-1, a gene encoding a Wnt antagonist, responds to DNA damage and its overexpression sensitizes brain tumor cells to apoptosis following alkylation damage of DNA. Oncogene 2002, 21, 878-889. [CrossRef] [PubMed]

25. Zhao, Y.P.; Wang, W.; Wang, X.H.; Xu, Y.; Wang, Y.; Dong, Z.F.; Zhang, J.J. Downregulation of serum DKK-1 predicts poor prognosis in patients with papillary thyroid cancer. Genet. Mol. Res. 2015, 14, 18886-18894. [CrossRef] [PubMed]

26. Shi, X.D.; Yu, X.H.; Wu, W.R.; Xu, X.L.; Wang, J.Y.; Xu, L.B.; Zhang, R.; Liu, C. Dickkopf-1 expression is associated with tumorigenity and lymphatic metastasis in human hilar cholangiocarcinoma. Oncotarget 2016, 7, 70378-70387. [CrossRef] [PubMed]

27. Kim, B.K.; Lee, J.W.; Park, P.J.; Shin, Y.S.; Lee, W.Y.; Lee, K.A.; Ye, S.; Hyun, H.; Kang, K.N.; Yeo, D.; et al. The multiplex bead array approach to identifying serum biomarkers associated with breast cancer. Breast Cancer Res. 2009, 11, R22. [CrossRef] [PubMed]

28. Sheng, S.L.; Huang, G.; Yu, B.; Qin, W.X. Clinical significance and prognostic value of serum Dickkopf-1 concentrations in patients with lung cancer. Clin. Chem. 2009, 55, 1656-1664. [CrossRef]

29. Huang, L.; Chen, W.; Liang, P.; Hu, W.; Zhang, K.; Shen, S.; Chen, J.; Zhang, Z.; Chen, B.; Han, Y.; et al. Serum CYFRA 21-1 in Biliary Tract Cancers: A Reliable Biomarker for Gallbladder Carcinoma and Intrahepatic Cholangiocarcinoma. Dig. Dis. Sci. 2015, 60, 1273-1283. [CrossRef]

30. Leelawat, K.; Narong, S.; Wannaprasert, J.; Ratanashu-ek, T. Prospective study of MMP7 serum levels in the diagnosis of cholangiocarcinoma. World J. Gastroenterol. 2010, 16, 4697-4703. [CrossRef]

31. Voigtlander, T.; Gupta, S.K.; Thum, S.; Fendrich, J.; Manns, M.P.; Lankisch, T.O.; Thum, T. MicroRNAs in Serum and Bile of Patients with Primary Sclerosing Cholangitis and/or Cholangiocarcinoma. PLoS ONE 2015, 10, e0139305. [CrossRef]

32. Lee, H.S.; Lee, H.E.; Park, D.J.; Kim, H.H.; Kim, W.H.; Park, K.U. Clinical significance of serum and tissue Dickkopf-1 levels in patients with gastric cancer. Clin. Chim. Acta 2012, 413, 1753-1760. [CrossRef]

33. Banales, J.M.; Cardinale, V.; Carpino, G.; Marzioni, M.; Andersen, J.B.; Invernizzi, P.; Lind, G.E.; Folseraas, T.; Forbes, S.J.; Fouassier, L.; et al. Expert consensus document: Cholangiocarcinoma: Current knowledge and future perspectives consensus statement from the European Network for the Study of Cholangiocarcinoma (ENS-CCA). Nat. Rev. Gastroenterol. Hepatol. 2016, 13, 261-280. [CrossRef]

34. Carotenuto, P.; Fassan, M.; Pandolfo, R.; Lampis, A.; Vicentini, C.; Cascione, L.; Paulus-Hock, V.; Boulter, L.; Guest, R.; Quagliata, L.; et al. Wnt signalling modulates transcribed-ultraconserved regions in hepatobiliary cancers. Gut 2017, 66, 1268-1277. [CrossRef]

35. Boulter, L.; Guest, R.V.; Kendall, T.J.; Wilson, D.H.; Wojtacha, D.; Robson, A.J.; Ridgway, R.A.; Samuel, K.; Van Rooijen, N.; Barry, S.T.; et al. WNT signaling drives cholangiocarcinoma growth and can be pharmacologically inhibited. J. Clin. Investig. 2015, 125, 1269-1285. [CrossRef]

36. Timmer, M.R.; Beuers, U.; Fockens, P.; Ponsioen, C.Y.; Rauws, E.A.; Wang, K.K.; Krishnadath, K.K. Genetic and epigenetic abnormalities in primary sclerosing cholangitis-associated cholangiocarcinoma. Inflamm. Bowel Dis. 2013, 19, $1789-1797$. [CrossRef]

37. Ghidini, M.; Pizzo, C.; Botticelli, A.; Hahne, J.C.; Passalacqua, R.; Tomasello, G.; Petrelli, F. Biliary tract cancer: Current challenges and future prospects. Cancer Manag. Res. 2019, 11, 379-388. [CrossRef] [PubMed]

38. Zong, Y.; Stanger, B.Z. Molecular mechanisms of bile duct development. Int. J. Biochem. Cell Biol. 2011, 43, 257-264. [CrossRef]

39. Rimland, C.A.; Tilson, S.G.; Morell, C.M.; Tomaz, R.A.; Lu, W.Y.; Adams, S.E.; Georgakopoulos, N.; Otaizo-Carrasquero, F.; Myers, T.G.; Ferdinand, J.R.; et al. Regional differences in human biliary tissues and corresponding in vitro derived organoids. Hepatology 2021, 73, 247-267. [CrossRef]

40. Chen, W.; Liang, J.; Huang, L.; Cai, J.; Lei, Y.; Lai, J.; Liang, L.; Zhang, K. Characterizing the activation of the Wnt signaling pathway in hilar cholangiocarcinoma using a tissue microarray approach. Eur. J. Histochem. 2016, 60, 2536. [CrossRef]

41. Madariaga, J.R.; Iwatsuki, S.; Todo, S.; Lee, R.G.; Irish, W.; Starzl, T.E. Liver resection for hilar and peripheral cholangiocarcinomas: A study of 62 cases. Ann. Surg. 1998, 227, 70-79. [CrossRef] 